\title{
REALISIERUNG EINES BISTABILEN FEUCHTESENSORSCHALTERS MIT SCHALTHYSTERESE
}

\author{
Nikolai Gulnizkii ${ }^{1}$, Gerald Gerlach ${ }^{1}$ \\ ${ }^{1} T U$ Dresden, Institut für Festkörperelektronik, Mommsenstraße 15, 01062 Dresden, Deutschland
}

\section{Zusammenfassung}

Hydrogel-basierte Sensorschalter können zur Inline-Überwachung der Luftfeuchte in Gebäuden, Containern, der Lebensmittelindustrie etc. eingesetzt werden. Der wesentliche Unterschied leistungsloser Feuchtesensoren zu gängigen Feuchtesensoren ist, dass für den Sensorbetrieb selbst keine Betriebsspannung benötigt wird. Auf diese Weise ermöglichen solche Sensorschalter eine deutliche Vereinfachung des Aufbaus. Das Prinzip basiert darauf, dass das Schalten des Mikroschalters beim Überschreiten eines Schwellwertes durch die Luftfeuchte selbst ausgelöst wird, so dass dafür keine elektrische Energie benötigt wird. Dies erfolgt durch einen Bimorphbalken mit einer Hydrogelschicht, die durch Änderungen der Luftfeuchtigkeit quillt bzw. entquillt. Um ein sicheres Schalten zu gewährleisten, muss die mechanische Struktur eine Schalthysterese aufweisen. Dabei kann die Hysterese durch ein belastungsabhängiges Ausbeulen einer axial belasteten Struktur hervorgerufen werden. In diesem Beitrag soll ein solcher Hydrogel-basierter Sensorschalter mit Schalthysterese für die Feuchte vorgestellt werden.

Keywords: Hydrogel, Hysterese, Mikroschalter, leistungslos, Bimorph-Effekt

\section{Motivation}

Die wachsende informationstechnische Vernetzung in der industriellen Produktion führt zu einer zunehmenden Nachfrage nach Sensorsystemen, die die Messwerterfassung, die Signalaufbereitung und die Signalverarbeitung vereinen. Solche Sensorsysteme erzielten in der Sensor- und Messtechnikindustrie in den vergangenen Jahrzehnten ein überdurchschnittliches Wachstum von über 6\% pro Jahr [1]. Dabei sind die wichtigsten Messgrößen die Temperatur und die Feuchtigkeit. Allerdings werden $70 \%$ aller Sensoren in der Prozesskontrolle und mehr als $90 \%$ in der Gebäudeüberwachung als Schwellwertschalter eingesetzt. In [2] wurde ein neuartiger Ansatz für einen leistungslosen Feuchteschwellwertschalter vorgeschlagen, der auf dem BIZEPS-Prinzip (binary zeropower sensor) basiert [3]. Bei diesem Prinzip wird die Schaltenergie direkt aus der Messgröße entnommen. In dem hier vorgestellten Beispiel wird dazu das feuchteabhängige Quellen eines Hydrogels genutzt, das Teil einer Bimorph-Biegeplatte ist. In dieser Arbeit soll ein vereinfachtes Sensorkonzept zur Beschreibung des Schaltvorgangs entwickelt und entsprechend ein Sensorschalter mit einer Schalthysterese realisiert werden.

\section{Funktionsweise}

Abb. 1 zeigt beispielhaft den Aufbau und das Funktionsprinzip eines solchen Feuchteschwellwertschalters [3][4].

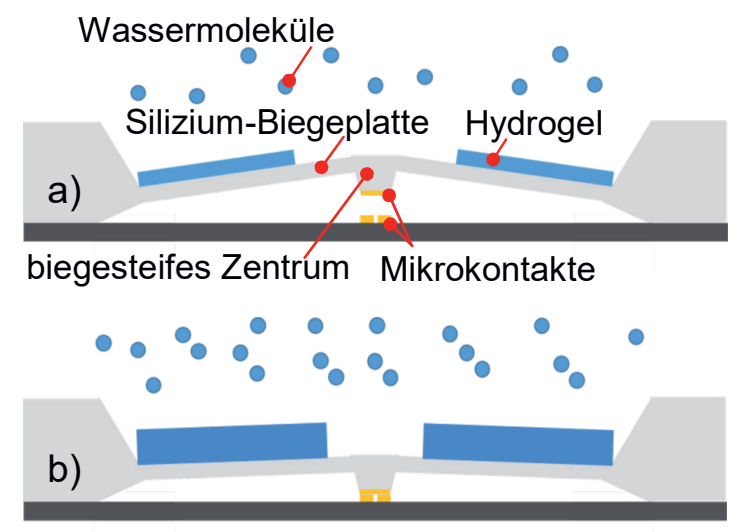

Abb. 1: Sensorschalter zur Feuchtemessung, bestehend aus einer BimorphBiegeplatte, einem biegesteifen Zentrum, einer strukturierten Hydrogelschicht und Mikrokontakten, a) geöffnet und b) geschlossen.

Er besitzt eine Biegeplatte, in deren Mitte sich ein biegesteifes Zentrum, das zur lokalen Versteifung der Biegeplatte führt, befindet. Es gehört zum schließenden bzw. zum öffnenden 
Kontaktpaar und stellt sicher, dass der Mikrokontakt auch bei Auslenkung der Biegeplatte eben bleibt und damit eine sichere Kontaktierung der Mikrokontakte erfolgt. Das biegesteife Zentrum der Biegeplatte wird durch die feuchteabhängige Quellung des Hydrogels auf der Hydrogel-beschichteten Siliziumplatte ausgelenkt. Der Quellzustand des Hydrogels hängt von der Absorption der Wassermoleküle aus der Luft, d.h. der Luftfeuchte der Umgebungsluft, ab.

Beim Quellen leistet das Hydrogel mechanische Arbeit und deformiert so die Biegeplatte. Das bedeutet, dass die kinetische Energie aus der Umgebung entnommen und der Sensor damit autonom und ohne elektrische Energieversorgung betrieben wird. Da es sich bei der Quellung und Entquellung des Hydrogels um einen reversiblen Prozess handelt, kann der Sensorschalter die Mikrokontakte sowohl öffnen als auch schließen.

Um ein sicheres Schalten des elektrischen Mikrokontakts zu gewährleisten, müssen ein oszillierendes Öffnen bzw. Schließen der Kontakte im Schaltpunkt und die Ausbildung von Lichtbögen vermieden werden. Dies kann durch eine Schalthysterese im Sensorschalter, der zwischen zwei stabilen Zuständen schaltet, erreicht werden [5]. In der hier vorgestellten Lösung wird dazu als Verformungskörper ein Biegebalken verwendet, auf den eine axiale Druckkraft aufgeprägt wird.

\section{Modell zur Berechnung der Balkendurchbiegung in Abhängigkeit vom Beschichtungsgrad}

Für das bessere physikalische Verständnis soll im Folgenden statt der Platte von Abb. 1 ein zweiseitig eingespannter Biegebalken betrachtete werden. Zur Berechnung der Durchbiegung des Biegebalkens in Abhängigkeit vom Beschichtungsgrad (Verhältnis der beschichteten zur gesamten Fläche) wird ein einfaches mechanisches Modell verwendet, das auf der Balkentheorie für monomorphe und bimorphe Balken basiert [6][7]. Abbildung 2 zeigt einen beidseitig eingespannten, am Balkenrand mit Hydrogel beschichteten Balkens. Der Balken aus Stahlblech besteht aus dem mit Hydrogel beschichteten Teil mit der längenabhängigen Nachgiebigkeit $n_{B}$ und dem unbeschichteten Teil mit der ebenfalls längenabhängigen Nachgiebigkeit $n_{P}$. Dabei entspricht $I_{P}$ der Länge des Biegebalkens, $h$ der Balkendicke und $b$ der Balkenbreite. Da das Hydrogel sehr weich ist, wird die jeweilige Nachgiebigkeit durch die Elastizität des Blechs bestimmt:

$$
\begin{aligned}
& n_{B}=12 \frac{l_{B}}{E b h^{3}}, \\
& n_{P}=12 \frac{l_{P}}{E b h^{3}} .
\end{aligned}
$$

Der Bimorph aus Hydrogel und Blech wirkt bei Quellung des Hydrogels als Momentenquelle:

$$
M_{r . F .}=\varepsilon_{r . F .} \frac{b E_{2} h_{2}\left(h_{1}+h_{2}\right)}{2\left(1+\frac{E_{2} h_{2}}{E_{1} h_{1}}\right)}
$$

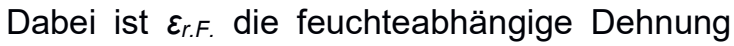
des Hydrogels.

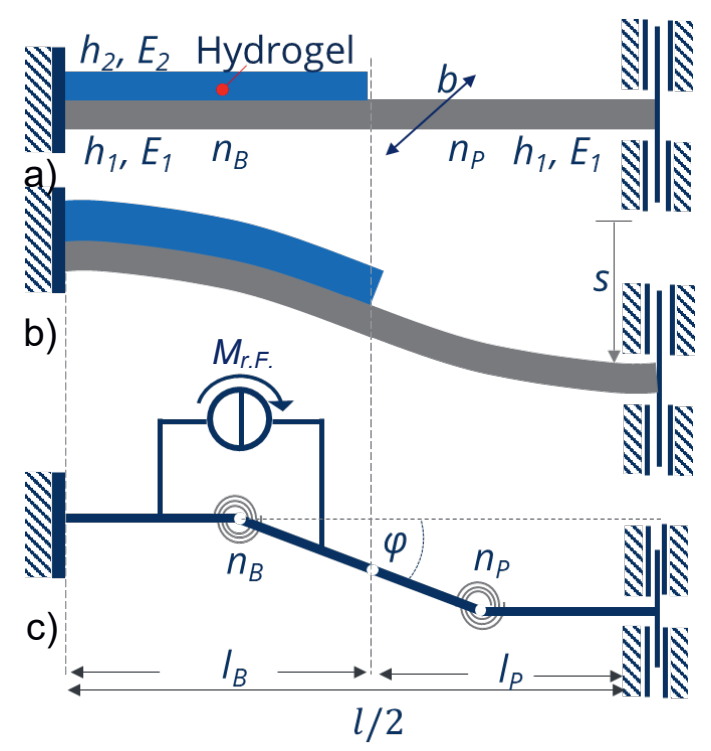

Abb. 2: Modell eines balkenförmigen Sensorschalters gemäß Abb. 1. a) schematische Darstellung des halben Sensorschalters, b) ausgelenkter Sensorschalter bei Hydrogelquellung und c) korrespondierendes mechanisches Modell. $n_{B}, \quad n_{P}$ längenabhängige Nachgiebigkeiten der Biegebalken mit und ohne Hydrogelschicht, I/2 Balkenlänge, I Länge des mit Hydrogel beschichteten Balkens, s Auslenkung, $\varphi$ Auslenkungswinkel, $M_{\text {r.F. }} \quad$ feuchteinduzierte Momentenquelle, b Breite, $h$ Dicke, Indizes: 1 Substrat, 2 Hydrogel, P unbeschichtet, B Hydrogel bzw. mit Hydrogel beschichtet.

Mit der Annahme, dass die Hydrogelschicht und der Biegebalken vergleichbar dick sind ( $h_{1} \approx h_{2}$ ), ergibt sich aus GI. (3):

$$
M_{r . F .}=b E_{2} h_{2} \varepsilon_{r . F}
$$


und für die Auslenkung des Biegebalkens:

$$
s=\frac{l}{2} \varphi=\frac{l}{2} n M_{r . F .},
$$

wobei die Gesamtnachgiebigkeit

$$
n=\frac{n_{B} n_{P}}{n_{B}+n_{P}}
$$

ist.

Mit den Gleichungen (1) und (2) folgt für die Auslenkung:

$$
s=6 \frac{E_{2} l_{B}}{E_{1} h} \frac{l_{B}}{l}\left(1-\frac{l_{B}}{l}\right) \varepsilon_{r . F .} .
$$

Damit hängt die Auslenkung maßgeblich vom E-Modul $E_{2}$ des Hydrogels, von den geometrischen Abmessungen der Hydrogelschicht, von der feuchteabhängigen Quelldehnung $\varepsilon_{\text {r.F. }}$ und vom Beschichtungsgrad IB $/ \mathrm{ab}$.

Aus Gleichung (7) folgt, dass eine große Durchbiegung

- durch ein großes Verhältnis $E_{2} / E_{1}$ der E-Moduln von Hydrogel und Substrat (Biegebalken: Stahl),

- durch dünne Biegebalken und

- durch große Quelldehnungen $\varepsilon_{\text {r.F. bzw. }}$ Ausdehnungskoeffizienten $\alpha_{\text {r.F. }}$ in Folge der relativen Feuchte

erreicht werde kann.

Eine maximale Durchbiegung $s_{\max }$ wird für dieses vereinfachte Modell erzielt, wenn das Verhältnis $I_{B} / l$ dem Wert 0,5 entspricht. Aus den Gleichungen (6) und (7) kann für komplizierte Anordnungen aber auch abgeleitet werden, dass die Auslenkung dann maximal wird, wenn die Nachgiebigkeiten $n_{B}$ und $n_{P}$ des beschichteten und unbeschichteten Teils gleich groß sind.

\section{Balkenmodell mit Axialkraft}

Für technische Anwendungen muss der Schaltvorgang zum Öffnen und Schließen von Mikrokontakten sehr schnell erfolgen. Dies wird hier durch eine axiale Krafteinwirkung, die zu einer Vorauslenkung des Balkens führt, realisiert. Abbildung 3 zeigt das mechanische Modell eines vorausgelenkten Balkens mit axialer Krafteinwirkung. Der Vorauslenkungswinkel $\varphi_{0}$ hängt hierbei von der längenabhängigen Nachgiebigkeit $n$ aus GI. (6) und dem Betrag der Kraft $F_{A}$ ab.

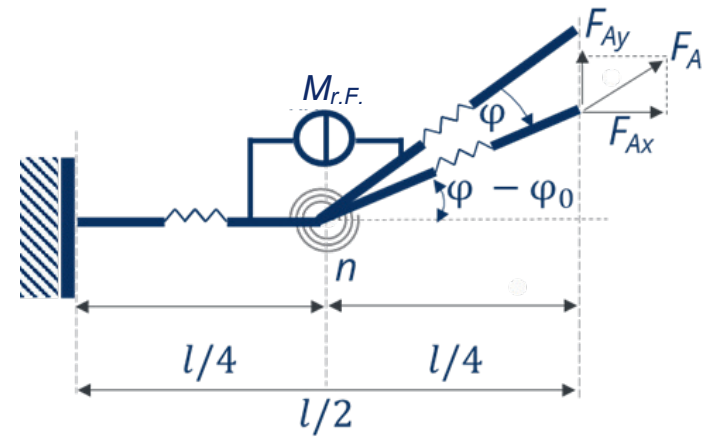

Abb. 3: Mechanisches Modell zur Beschreibung eines durch die Axialkraft $F_{A} \quad$ vorausgelenkten Balkens mit der Länge I/2, der Nachgiebigkeit $n$ und dem Auslenkungswinkel $\varphi_{0}$. Dargestellt ist eine Balkenhälfte. Das Biegemoment $M_{\text {r.F. entspricht hierbei einer externen }}$ Momentenquelle. $F_{A x}$ und $F_{A y}$ sind die vektoriellen Komponenten von $F_{A}$.

Die Kraft $F_{A}$ wird hierbei vektoriell in zwei Kraftkomponenten $F_{A x}$ und $F_{A y}$ zerlegt. Die Auslenkung des Balkens durch die externe Momentenquelle wird durch den Auslenkungswinkel $\varphi$ und die Gesamtauslenkung durch den Winkel $\varphi=\varphi+\varphi_{0}$ beschrieben. Damit lässt sich für kleine Auslenkungswinkel die Momentengleichung herleiten:

$M_{r . F .}-F_{A} \frac{l}{4}\left(\varphi-\varphi_{0}\right)+F_{A}\left(\varphi-\varphi_{0}\right)^{3}=0$,

sodass die Auslenkung des Balkens von der Balkengeometrie, den Materialparametern und der einwirkenden Axialkraft abhängt. In GI. (8) weist die Beziehung zwischen Winkel $\varphi$ und damit Auslenkung $s$ am rechten Balkenende in Abb. 3 und dem zum Hyrogelquellen proportionalen Biegemoment $M_{\text {r.F. neben dem }}$ linearen Glied auch ein kubisches Glied auf. Dieses führt zu der in Abb. 4 dargestellten SKurve. Steigt die Feuchte an und übersteigt dabei $\varphi_{\text {r.F.oben, ändert sich die Auslenkung }}$ plötzlich von $s_{1}$ zu $s_{2}$. Sinkt die Feuchte soweit, dass sie $\varphi_{\text {r.F.unten }}$ unterschreitet, ändert sich die Auslenkung plötzlich von $s_{3}$ auf $s_{4}$ und der Schalter öffnet sich. 


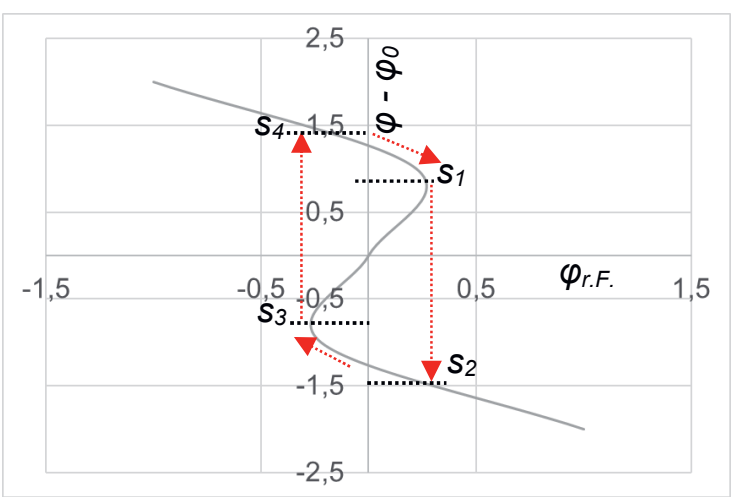

Abb. 4: Berechnete S-Kurve aus GI. (8). Die Schaltpunkte sind mit den vertikal verlaufenden Pfeilen gekennzeichnet.

\section{Aufbau und Herstellung}

Um die Funktionsweise des hier vorgestellten Feuchtesensorschalters zu demonstrieren, wird der Aufbau von Abb. 5 verwendet, bei dem auf den Balken $\left(n_{P}\right)$ eine axiale Kraft $F_{A}$ aufgeprägt und damit die Schalthysterese erreicht wird.

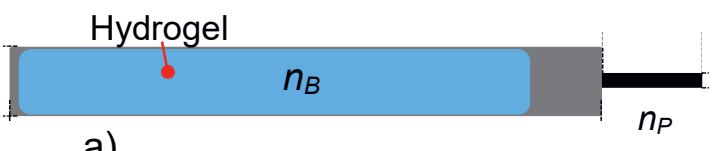

a)

Halterung Axialkraft

Halterung

b) Auslenkung $s$ Stellschraube

Abb. 5: Balkenbasierter Sensorschalter mit Schalthysterese: a) Draufsicht, b) Querschnitt.

Der Balken besteht aus einem breiten und dickeren, Hydrogel-beschichteten Teil mit der Nachgiebigkeit $n_{B}$ und einem schmaleren Teil mit der Nachgiebigkeit $n_{P}$ (Material- und Geometriedaten siehe Tabelle 1).

Tab. 1: Geometrie- und Materialparameter.

\begin{tabular}{|l|l|l|}
\hline Parameter & Blech $n_{B} \mid n_{P}$ & Hydrogel \\
\hline Länge $[\mathrm{mm}]$ & $55 \mid 7$ & 50 \\
\hline Breite $[\mathrm{mm}]$ & $10 \mid 1$ & 10 \\
\hline Dicke $[\mathrm{mm}]$ & $0,03 \mid 0,01$ & 0,03 \\
\hline E-Modul & 210000 & $3500(5 \%$ r.F. $)$ \\
{$\left[\mathrm{N} / \mathrm{mm}^{2}\right]$} & & $300(90 \%$ r.F. $)$ \\
\hline
\end{tabular}

Der Balken aus Stahlblech (DC01) wird dabei durch Zuschneiden gefertigt.

Für eine ausreichende Haftung der $0,03 \mathrm{~mm}$ dicken Hydrogelschicht wurde die Blechoberfläche chemisch gereinigt, plasmaaktiviert und mit einem Haftvermittler vorbehandelt. $\mathrm{Zu}$ Beginn wurde der
Biegebalken für 15 Minuten in einer Lösung aus destilliertem $\mathrm{H}_{2} \mathrm{O}, 25 \%$ Ammoniak und $25 \%$ Peroxid $(2: 1: 1)$ bei $85{ }^{\circ} \mathrm{C}$ gereinigt. Danach wurde die Oberfläche mit Sauerstoffplasma (VTD: DREVA 450) bei einem Druck von $15 \mathrm{~Pa}$ bei $400 \mathrm{~W}$ für $5 \mathrm{~min}$ aktiviert. Anschließend wurden $3 \mathrm{ml}$ einer Haftvermittlerlösung, bestehend aus $0,9 \mathrm{ml}$ Haftvermittler (3-Aminopropyl)Triethoxysilan (APTES) (Carl Roth) und $99 \mathrm{ml} \mathrm{H}_{2} \mathrm{O}$ auf die plasmabehandelte Oberfläche aufgebracht und 10 Minuten im Ofen bei $100^{\circ} \mathrm{C}$ geheizt [8]. Für die Hydrogelstrukturierung wurden $5 \mathrm{ml}$ einer Polymerlösung aus $15 \mathrm{wt} \%$ Polyvinylalkohol (PVA, Molekularmasse $M w=89,000 \ldots$ $98,000)$ und $7.5 \mathrm{wt} \%$ Polyacrylsäure (PAS, $M w$ $=450,000)$ in einem Massenverhältnis von 4:1 auf die vorbehandelte Oberfläche pipettiert und für $6 \mathrm{~h}$ bei $22{ }^{\circ} \mathrm{C}$ getrocknet. Anschließend wurde die strukturierte Polymerschicht in einem Ofen bei $120{ }^{\circ} \mathrm{C}$ für 20 Minuten thermisch vernetzt [9] [10].

Beide Biegebalken $n_{B}$ und $n_{P}$ wurden mit Sekundenkleber miteinander verklebt. Die Blechstruktur wurde in einer Halterung fixiert und anschließend mit einer Stellschraube vorausgelenkt (Abb. 5).

\section{Messung der Schalthysterese am makroskopischen Sensorschalter}

Die Vorauslenkung wurde durch einen axialen Krafteintrag von 0,02 N mittels Verstellen einer Stellschraube erreicht. Zur Messung der Axialkraft wurde auf der gegenüberliegenden Seite der Befestigung ein Kraftmessgerät (SAUTER FC 10, FC Compact) eingesetzt.

Die Feuchte in der Feuchtekammer wurde dabei mittels eines Blasen-Systems variiert. Die Änderung der relativen Feuchte wurde mittels eines kapazitiven Feuchtesensors überwacht. Zum Nachweis der feuchteabhängigen Auslenkung der eingespannten Blechstruktur wurde eine CMOS-Kamera (HIKVISON USB3.0, Vision SXGA, monochrom) verwendet (Abb. 6). 


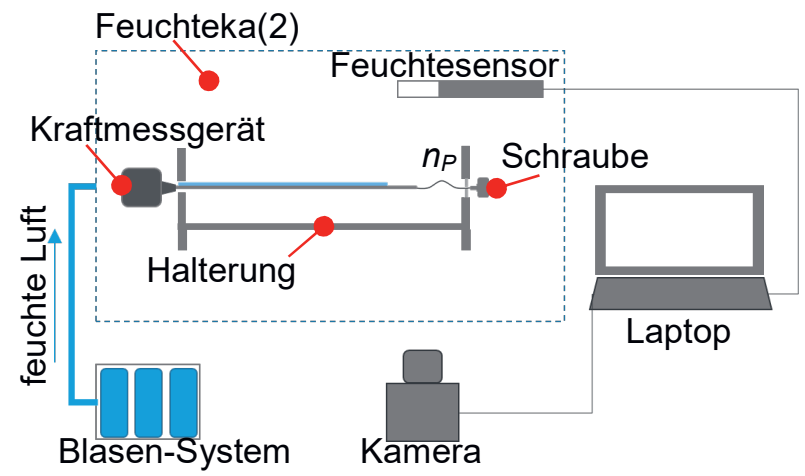

Abb. 6: Schematische
Messaufbaus, Darstellung des
Feuchtekammer, Fehend aus
Blasen-system, Kamera und
Sensorschalter.

\section{Messergebnisse und Diskussion}

Nachdem das Hydrogel auf einer Biegebalkenhälfte strukturiert wurde, wurden $n_{B}$ und $n_{P}$ am Balkenrand miteinander verklebt und in einer speziell für diese Anwendung hergestellten Halterung fixiert. Anschließend wurde durch das Verstellen der Stellschraube die Balkenstruktur einseitig axial belastet. Auf der gegenüberliegenden Seite der Stellschraube wurde die Axialkraft durch ein Kraftmessgerät überwacht. Nach Erreichen der Axialkraft von $0,02 \quad \mathrm{~N}$ wurde die feuchteabhängige Auslenkung des Balkens bestimmt. Abbildung 7 zeigt die Ergebnisse der Messung der feuchteabhängigen Auslenkung mit der resultierenden Schalthysterese und ihren zwei Schaltpunkten bei 58 \%r.F. und bei $75 \%$ r.F.

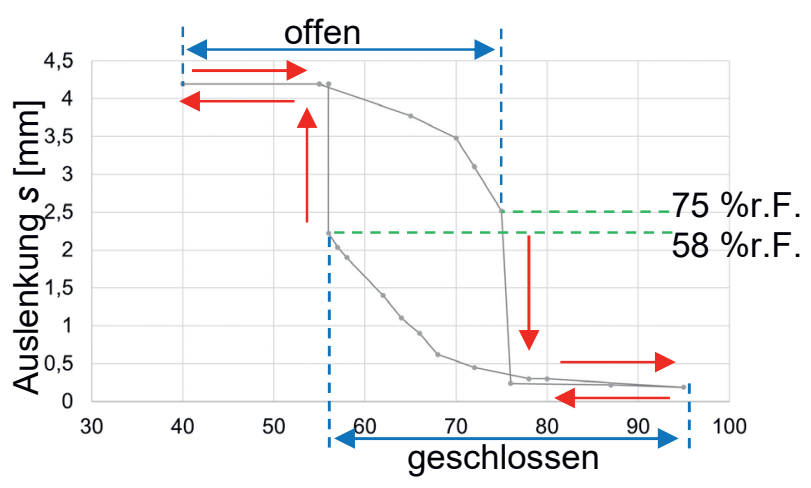

Relative Feuchte [\%]

Abb. 7: Auslenkung s der Biegefeder $n_{P}$ in Abhängigkeit von der relativen Feuchte mit den zwei Schaltpunkten bei $58 \%$ r.F. und $75 \%$ r.F.

\section{Zusammenfassung}

Zur Überwachung der Feuchte wurde ein leistungsloser Sensorschalter mit Hydrogel entwickelt. Es besteht aus einem Biegebalken, der teilweise mit Hydrogel beschichtet ist und der axial mit einer Druckkraft beaufschlagt wird, um die gewünschte Schalthysterese zu erreichen. Mit einem einfachen Mono/Bimorph-Balkenmodell wurde gezeigt, dass die erreichbare Auslenkung maximal wird, wenn die Nachgiebigkeit des beschichteten und des unbeschichteten Balkenteils gleich groß sind bzw. der Bedeckungsgrad etwa $50 \%$ beträgt. Mit der Axialkraft wird eine Vorauslenkung erzielt, sodass beim Quellen des Hydrogels ein Durchschlagen des Balkens und damit ein schnelles Schalten erreicht wird. Die Schalthysterese resultiert dabei aus der SKurve, die durch den kubischen Funktionsanteil der Auslenkungs-Feuchtekurve verursacht wird. Anhand eines Demonstrators konnte die prinzipielle Funktionsweise der Schalthysterese nachgewiesen werden.

\section{Literaturnachweis}

[1] AMA: Sector Information: Quarterreports 2017, http://www.amasensorik.de/en/association/sectorinformation/quarterreports-2017/ - (data from 08/14/2018)

[2] C. Bellmann, R. Sarwar, A. Steinke, T. Frank, H. F. Schlaak and G. Gerlach, Development of a humidity micro switch based on humidity sensitive polymers, 23rd Micromechanics and Microsystems Europe Workshop (MME), Ilmenau, Germany, (2012)

[3] T. Frank, A. Steinke, and G. Gerlach, Binary ZeroPower Sensors: an alternative solution for power-free energy-autonomous sensor systems, Microsystem Technologies 18(7-8), 1225-1231 (2012)

[4] C. Bellmann, Leistungslose Feuchteschwellwertschalter, Dissertation, TU Dresden, (2016)

[5] L.T. Chen, C. Y. Lee, W. H. Cheng, MEMSbased humidity sensor with integrated temperature compesation mechanism, Sensors and Actors A:147, 522-528, (2008)

[6] U. Marschner, G. Gerlach, E. Starke, A. Lenk, Equivalent circuit models of two-layer flexure beams with excitation by temperature, humidity, pressure, piezoelectric or piezomagnetic interactions, Vol.3, Page 187-211, JSSS (2014)

[7] H. Balke, Einführung in die Technische Mechanik, 3. Auflage, Springer-Verlag Berlin Heidelberg 2010

[8] N. Gartmann, C. Schütze, H. Ritter, D. Brühwiler, The effect of water on the functionalization of mesoporous silica with 3aminopropyltriethoxysilane, Journal of Physical Chemistry Letters 1, 379-382, (2010)

[9] J. Jose, F. Shehzad, M. A. Al-Harthi, Preparation method and physical, mechanical, thermal characterization of poly (vinyl alcohol)/poly(acrylic acid) blends, Polymer Bulletin 71(11), 2787-2802, (2014) 
[10] K. F. Arndt, A. Richter, S. Ludwig, J.

Zimmermann, J. Kessler, D. Kuckling and H. J.

Adler, Poly (vinyl alcohol)/poly (acrylic acid)

hydrogels, FT-IR spectroscopic characterization

of crosslinking reaction and work at transition point, Acta Polymerica 50(11-12), 383-390,

(1999) 